\title{
Cálculo numérico de la matriz de flexibilidades de vigas de sección variable, con elementos finitos
}

\author{
Numerical computation of the flexibility matrix of variable \\ cross section beams, with finite elements
}

Jaime Retama Velasco

Facultad de Estudios Superiores Aragón Universidad Nacional Autónoma de México
Ricardo Heras Cruz Facultad de Estudios Superiores Aragón Universidad Nacional Autónoma de México

\section{Resumen}

En este trabajo se derivan las propiedades de flexibilidad de elementos de una viga de sección variable, mediante la aplicación del segundo teorema de Castigliano, considerando la energía complementaria de deformación por flexión y cortante. Se propone la integración de los coeficientes de flexibilidad de forma numérica, por medio de una discretización del dominio, con elementos finitos rectangulares de primer orden. Al final, el método de cálculo propuesto se aplicará a la solución de una viga ahusada, discretizada con un máximo de cinco de elementos finitos. Se muestra que dicho procedimiento es general y se puede aplicar a vigas de sección variable, cuya sección transversal sea compleja, lo que permitirá obtener soluciones numéricas satisfactorias con tres elementos finitos para viga con una relación peralte-longitud del orden de diez.

\section{Palabras clave:}

Integración numérica, elemento finito, vigas de sección variable, teoría de vigas.

\begin{abstract}
In this work, the flexibility properties of variable cross section beams are derived, through the application of the second theorem of Castigliano; considering the complementary energy by bending and share forces. To perform the integration of the flexibility coefficients, a numerical method, which considers the discretization of the beam domain with first order rectangular finite elements, in conjunction with the Gauss rule, is proposed. At the end of the work, the proposed method is applied to a tapered beam that has been discretized with a maximum of five finite elements. It is shown that the method is general, and that it can be applied to beams of variable section in which the cross section can be complex. The results shown that no more than 3 finite elements are needed to discretize the domain of beams in which, the ratio height-length is of the order of ten.
\end{abstract}

\section{Key words:}

Numerical integration, finite element, beams of variable cross section, beams theory.

Recibido: 12 de septiembre de 2019 Aceptado: 10 de diciembre de 2019

https://doi.org/10.22201/fesa.rdp.2020.1.03 


\section{Introducción}

El uso de elementos estructurales de sección variable, no-prismáticos, se ha extendido en la práctica de la ingeniería civil, para la construcción de diferentes obras de infraestructura: puentes, edificios, estadios, entre otras, porque permite optimizar las secciones, minimizando el peso, sin afectar su capacidad estructural que le ayuda a resistir las cargas que soporta.

Este tipo de vigas se puede clasificar en dos grupos: las vigas ahusadas (en inglés, tapered beams), Figura 1, en las que $x$ y $y$ son ejes de simetría; $y$ las vigas acarteladas (en inglés, haunched beams), Figura 2, en las que sólo el eje $y$ es simétrico (Alemdar, 2011; Attarnejad, Shahba y Jandaghi, 2011; Beltempo, Balduzzi, Alfano y Auricchio, 2015).

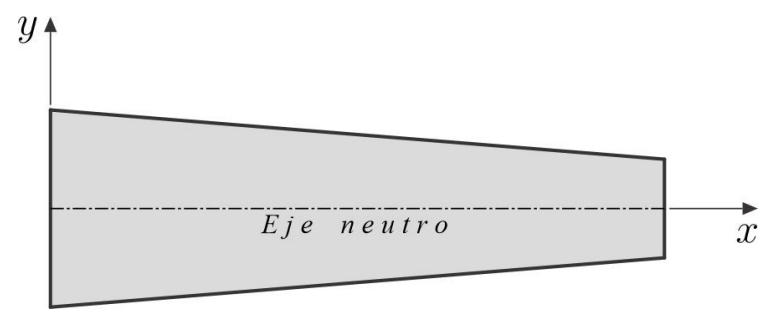

Figura 1. Viga ahusada. Elaboración: Jaime Retama Velasco.

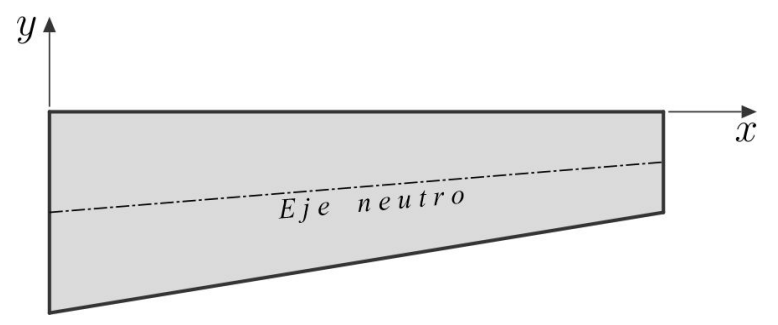

Figura 2. Viga acartelada.

Elaboración Jaime Retama Velasco.

Hoy en día, se cuenta con muchas formulaciones numéricas que consideran el efecto de variación de la sección transversal en toda la longitud de una viga, en sus propiedades de rigidez y flexibilidad; sin embargo, no es sencillo encontrar un modelo que ofrezca resultados exactos, por lo que el modelado numérico de este problema sigue siendo un tema de estudio en diferentes áreas de la ingeniería (Alemdar y White, 2005; Alemdar, 2011; Attarnejad et al., 2011; Eisenberger, 1991; Balduzzi, Morganti, Auricchio y Reali, 2017; Beltempo et al., 2015).
Diferentes autores han utilizado polinomios, funciones de Green, series de Taylor y el método de Frobenius para obtener una solución aproximada de las ecuaciones diferenciales que rigen el comportamiento de una viga (Eisenberger, 1990; Banerjee y Williams, 1986; Eisenberger, 1994; Zhang, Zhou, Xv y Lu, 2011; Veiskarami y Pourzeynali, 2012). Esto ha permitido llegar a soluciones aproximadas en puntos discretos de la viga y, al combinarse con el método de los elementos finitos, es posible obtener funciones de forma a partir de las ecuaciones diferenciales.

Otro método de uso generalizado por diversos autores es el de las flexibilidades, o de las fuerzas (Alemdar y White, 2005; Alemdar 2011; Attarnejad et al., 2011), el cual satisface el equilibrio en cualquier punto a lo largo del elemento; por ello, se le considera un método exacto de análisis estructural (Przemieniecki, 1985; Sack, 1994; Gallagher, 1975); la aplicación de este procedimiento implica la integración de una función en el dominio de la viga; para secciones complejas, es preferible integrar con un método numérico.

En este trabajo, se emplea el método de las fuerzas para calcular los coeficientes de flexibilidades de vigas de sección variable, y su integración numérica mediante la regla de Gauss. Para ello, se recurre a una discretización del volumen de la viga por medio de elementos finitos en dos dimensiones, lo que ayudará a minimizar el error de integración asociado a la aproximación numérica, a medida que se aumenta el número de elementos (Pilkey, 2002; Carrera, Giunta y Petrolo, 2011).

\section{Teoría de vigas}

La teoría de vigas simplifica el problema de sólidos a un problema unidimensional; se considera que la longitud es mucho mayor a las dimensiones transversales de la viga; cargas que actúan son perpendiculares a su eje longitudinal, el cual coincide con su eje centroidal. Las ecuaciones que rigen el comportamiento de una viga permiten definir su cinemática de deformación y las fuerzas internas, a partir de la curva de deflexión. Hay dos construcciones teóricas fundamentales que ayudan a describir la cinemática de deformación de una viga: la teoría de vigas de Euler-Bernoulli y la teoría de vigas de Timoshenko (Wang et al., 2000).

De la Figura 3, para relaciones de longitud-peralte mayores o iguales a diez, se tiene que la viga se comporta de 
acuerdo con la teoría de Euler-Bernoulli, y para relaciones menores a diez, su comportamiento se apega más a la teoría de Timoshenko.

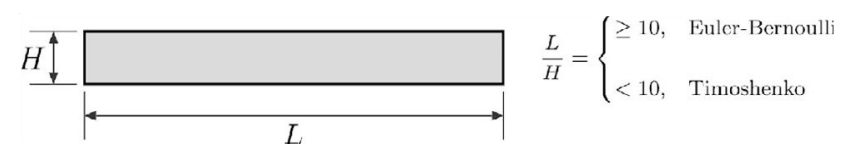

Figura 3. Relación entre longitud y peralte para vigas. Elaboración: Jaime Retama Velasco.

Al interpretar la Figura 4, se observa que conforme aumenta la relación longitud-peralte, la contribución del momento flexionante, en la flexión de la viga, se vuelve dominante; en el caso contrario, en vigas peraltadas, la flexión por fuerza cortante se incrementa, por lo que es necesario tomar en cuenta su contribución.

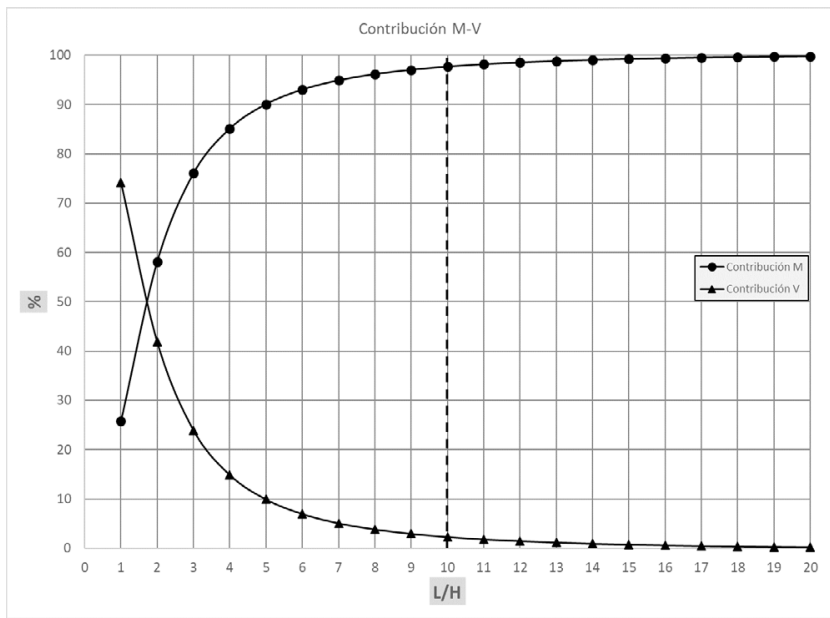

Figura 4. Contribución del momento y cortante en la flexión de vigas. Elaboración: Jaime Retama Velasco.

\subsection{Euler-Bernoulli}

En la teoría de vigas de Euler-Bernoulli se asume que las secciones planas y normales al eje axial de la viga, antes de la deformación, permanecen planas, y normales al eje después de la deformación, como se representa en la Figura 5. Para establecer las ecuaciones que gobiernan este problema, se considera que la función $v(x)$, que describe la deflexión del eje axial, satisface la ecuación diferencial de equilibrio (Reddy, 2019; Wang, Reddy y Lee, 2000; Carrera et al., 2011; Oden y Ripperger, 1981):

$\frac{d^{2}}{d x^{2}}\left(E I \frac{d^{2} v}{d x^{2}}\right)=q, \quad \rightarrow \quad 0<x<L$ donde $E$ es el módulo de elasticidad del material; $I$ es el momento de inercia de la sección transversal de la viga y $q$ define la carga transversal, distribuida de manera uniforme. Estas funciones dependen de $x$; la longitud de la viga está dada por $L$; la función de la curva elástica $v(x)$ debe satisfacer la ecuación de equilibrio 1 y las condiciones de frontera, esenciales y naturales. Para vigas en las que la sección transversal varía a lo largo de su longitud, la ecuación diferencial 1 se reescribe en la forma siguiente:

$E \frac{d^{2} I}{d x^{2}} \frac{d^{2} v}{d x^{2}}+E I \frac{d^{4} v}{d x^{2}}=q, \quad \rightarrow \quad 0<x<L$

y para el caso en que la sección transversal y el módulo de elasticidad sean constantes, se tiene:

$E I \frac{d^{4} v}{d x^{2}}=q, \quad \rightarrow \quad 0<x<L$

En ambos casos, sección variable y sección constante, la ecuación diferencial es no-homogénea y lineal de cuarto orden; para la sección variable los coeficientes varían en $x$, mientras que para la sección constante los coeficientes son constantes.

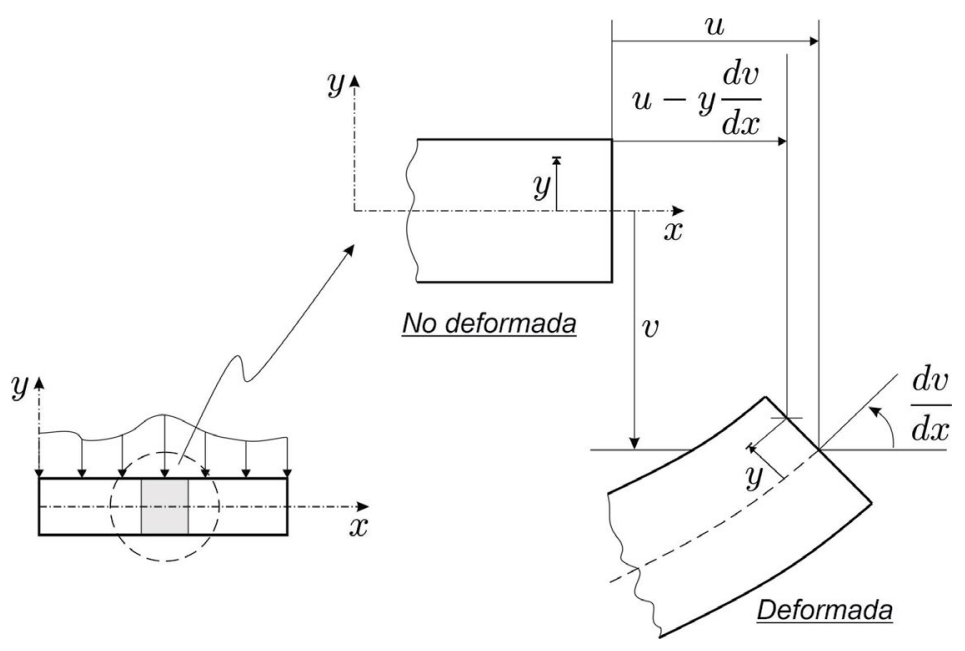

Figura 5. Cinemática de la viga de Euler-Bernoulli. Elaboración: Jaime Retama Velasco.

\section{2. Timoshenko}

En la teoría de vigas de Timoshenko, se asume que las secciones planas y normales al eje axial permanecen planas antes de la deformación, pero no normales al eje después 
de la deformación (Reddy, 2019; Wang et al., 2000; Oden y Ripperger, 1981), como en la Figura 6. Bajo esta consideración, las deformaciones cortantes son diferentes de cero, por lo tanto, la rotación de las secciones alrededor del eje axial no son la derivada de la función de deflexión $v(x)$. Para definir la rotación, se introduce la función independiente $\psi(x)$, por lo que el equilibrio de la viga está dado por:

$-\frac{d}{d x}\left[G A K_{S}\left(\psi+\frac{d v}{d x}\right)\right]=q$

$-\frac{d}{d x}\left(E I \frac{d \psi}{d x}\right)+G A \kappa\left(\psi+\frac{d v}{d x}\right)=0$

donde $G$ es el módulo de elasticidad cortante, $A$ es el área de la sección transversal de la viga y $k$ es el coeficiente de corrección por cortante, que toma en cuenta la diferencia entre el estado real parabólico de los esfuerzos por cortante y su idealización constante en la teoría de vigas.

La solución exacta de las ecuaciones diferenciales que gobiernan el problema de flexión en vigas, Euler-Bernoulli y Timoshenko, demanda operaciones matemáticas que pueden tornarse complejas, cuando las condiciones de geometría y de material varían a lo largo del eje axial de la viga. Por ello, es preferible llegar a esa solución mediante los métodos energéticos de la mecánica.

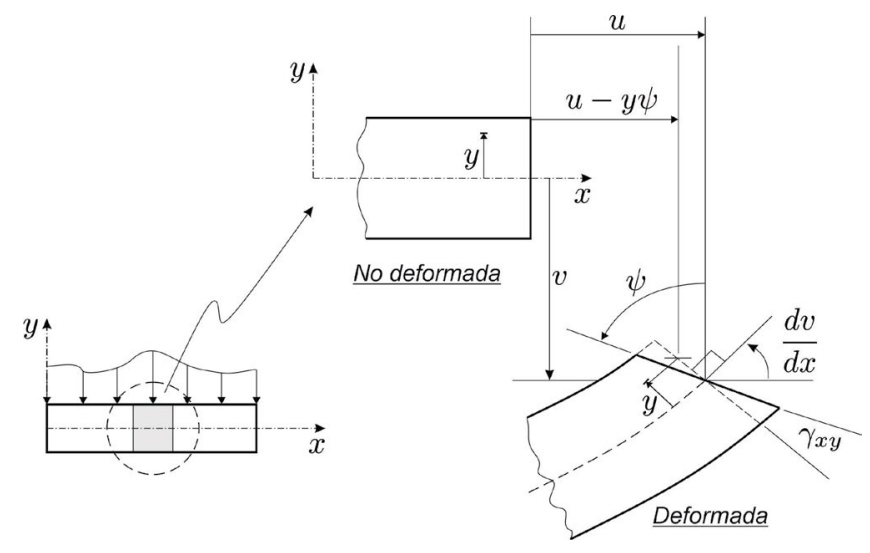

Figura 6. Cinemática de la viga de Timoshenko. Elaboración: Jaime Retama Velasco.

\section{Energía complementaria de deformación}

En la Figura 7 se muestra un elemento barra sujeto a la acción de los seis elementos mecánicos que se tienen en el espacio: fuerza normal, $N$; fuerza cortante, $V_{X}$; fuerza cortante, $V_{\dot{y}}$; momento flexionante, $M_{y}$; momento flexionante, $M_{z}$; y momento torsionante, $T$ (Oden y Ripperger, 1981).

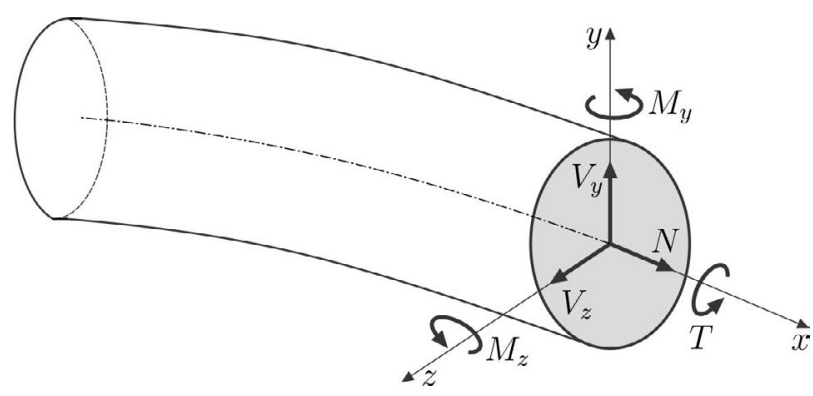

Figura 7. Barra en el espacio. Elaboración: Jaime Retama Velasco.

El problema de una barra se obtiene al establecer hipótesis que permiten simplificar la problemática tridimensional de los sólidos, a la de un elemento definido en una sola dimensión. Bajo esta condición, las variables de campo del problema se definen a lo largo del eje axial $x$ de la viga. Para la barra mostrada en la Figura 7, de un material elástico y lineal, la densidad de energía complementaria de deformación, área sobre la curva $\sigma$ - $\varepsilon$, se expresa como,

$$
u_{c}=\frac{1}{2} \sigma_{x} \varepsilon_{x}
$$

donde $\sigma_{x}$ y $\varepsilon_{x}$ son el esfuerzo y la deformación unitaria a lo largo del eje axial; para obtener la energía complementaria total de la barra, $U_{c}$, la ecuación 5 se integra en todo su volumen:

$$
U_{c}=\int_{V} u_{c} d V
$$

Al considerar los elementos mecánicos definidos en la Figura 7, y su efecto en la energía de deformación de la barra, la ecuación 6 se reescribe en su forma general (Ghali y Neville, 2017; Przemieniecki, 1985; Sack, 1994):

$U_{c}=\frac{1}{2} \int_{0}^{L}\left[\frac{N^{2}}{E A}+\frac{V_{y}^{2}}{G A} \kappa_{y}+\frac{V_{z}^{2}}{G A} \kappa_{z}+\frac{M_{y}^{2}}{E I_{y}}+\frac{M_{z}^{2}}{E I_{z}}+\frac{T^{2}}{G J_{0}}\right] d x$ 
Para el problema de un elemento barra definido en el plano de análisis $x-y$, la ecuación 7 se reduce a:

$$
U_{C}=\frac{1}{2} \int_{0}^{L}\left[\frac{N^{2}}{E A}+\frac{V_{y}^{2}}{G A} \kappa_{y}+\frac{M_{z}^{2}}{E I_{z}}\right] d x
$$

Las ecuaciones 7 y 8 son válidas para materiales con un comportamiento elástico y lineal; además, se consideran pequeñas deformaciones.

\section{Matriz de flexibilidades}

Para derivar la matriz de flexibilidades del elemento viga, se utiliza el segundo teorema de Castigliano, que establece: "la parcial de la función que define la energía complementaria de deformación [dada por las ecuaciones 7 u 8], respecto a una fuerza externa, $P_{i}$, es igual al desplazamiento $d_{i}$ en el punto de aplicación de la carga”, y se expresa como:

$$
\frac{\partial U_{c}}{\partial P_{i}}=d_{i}
$$

Aquí la fuerza externa $P_{i}$ se define de forma general para denotar una fuerza puntual o un momento. Lo mismo para el caso del desplazamiento, $d_{i}$, que puede ser una traslación o una rotación.

En la Figura 8, se muestra la viga isostática asociada para el caso de una viga de sección variable, tipo ahusada. Para obtener la matriz de flexibilidades, se aplica el segundo teorema de Castigliano, definido por la ecuación 9; se considera la energía de deformación por flexión más cortante.

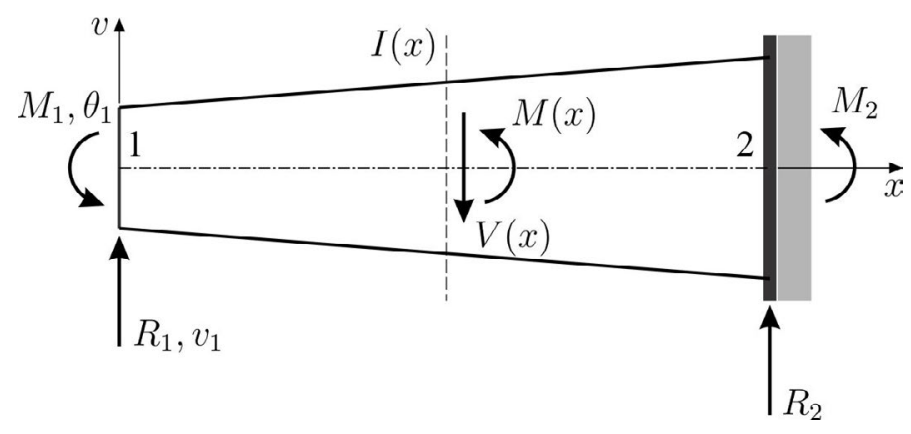

Figura 8. Viga ahusada isostática. Elaboración: Jaime Retama Velasco.

$$
U_{c}=\frac{1}{2} \int_{0}^{L}\left[\frac{V_{y}^{2}}{G A} \kappa_{y}+\frac{M_{z}^{2}}{E I_{z}}\right] d x
$$

Los coeficientes de flexibilidades se expresan como:

$$
\begin{aligned}
& f_{11}=\frac{1}{E} \int_{0}^{L} \frac{\left[x^{2}\right]}{I(x)} d x+\frac{k_{y}}{G} \int_{0}^{L} \frac{1}{A(x)} d x \\
& f_{12}=-\frac{1}{E} \int_{0}^{L} \frac{[x]}{I(x)} d x
\end{aligned}
$$

$$
f_{22}=\frac{1}{E} \int_{0}^{L} \frac{1}{I(x)} d x
$$

En las ecuaciones $11(\mathrm{a}, \mathrm{b}, \mathrm{c}$,$) se observa que la contri-$ bución de la energía de deformación por cortante sólo afecta el coeficiente de flexibilidad $f_{11}$; el resto de ellos son los mismos que cuando sólo se considera la energía por flexión.

\section{Elemento finito}

Para llevar a cabo la integración de los coeficientes de flexibilidades (dados por las ecuaciones 11) en todo el volumen de la barra, se hace una discretización del dominio por medio de elementos finitos en dos dimensiones (Pilkey, 2002). Este procedimiento permite simplificar la integración de los coeficientes de flexibilidades, cuando se tienen secciones complejas; se considera que el ancho de la sección transversal de la viga es constante, por lo que sólo se realiza una integral de área en el plano $x-y$; para ello, se utilizan elementos planos rectangulares de cuatro nodos de primer orden (Zienkiewicz, Taylor y Zhu, 2013; Rao, 2018; Fish y Belytschko, 2008).

\section{1. Elemento rectangular de primer orden}

La formulación de un elemento finito rectangular de cuatro nodos, empleado para discretizar el dominio de la viga, corresponde a un elemento con características geométricas particulares. En la Figura 9 se muestra el elemento finito, cuyas caras opuestas son paralelas y los ángulos internos son rectos. Las coordenadas nodales del 
elemento son $\left(x_{i}, y_{i}\right)$ para $i=1,2,3,4$. El espesor del elemento es el ancho, $B$, de la viga.

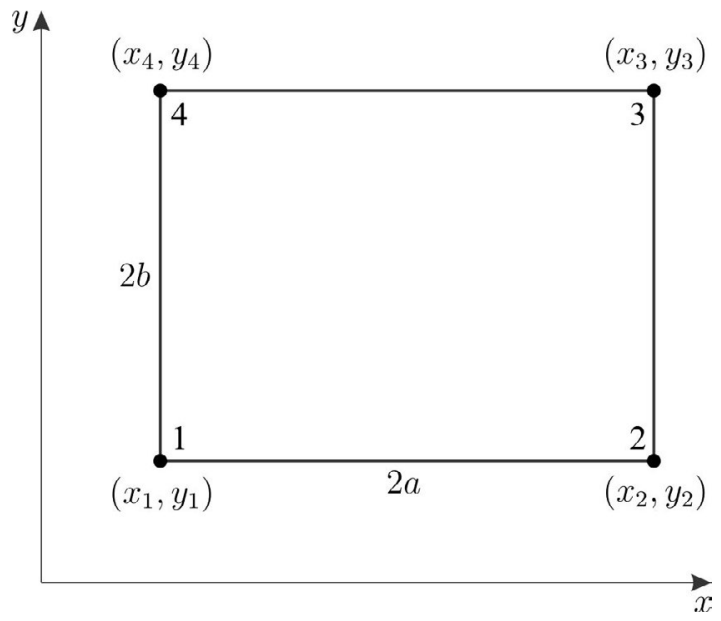

Figura 9. Elemento rectangular de primer orden. Elaboración: Jaime Retama Velasco.

Las dimensiones del elemento en el mundo real, Figura 9, son $2 a \times 2 b \times B$. La formulación del elemento finito se hace en el sistema de coordenadas naturales locales al elemento $(\xi, \eta)$, mundo padre, con su origen localizado en el centro de dicho elemento, Figura 10. Las dimensiones del elemento en el mundo padre son de $2 \times 2 \times B$.

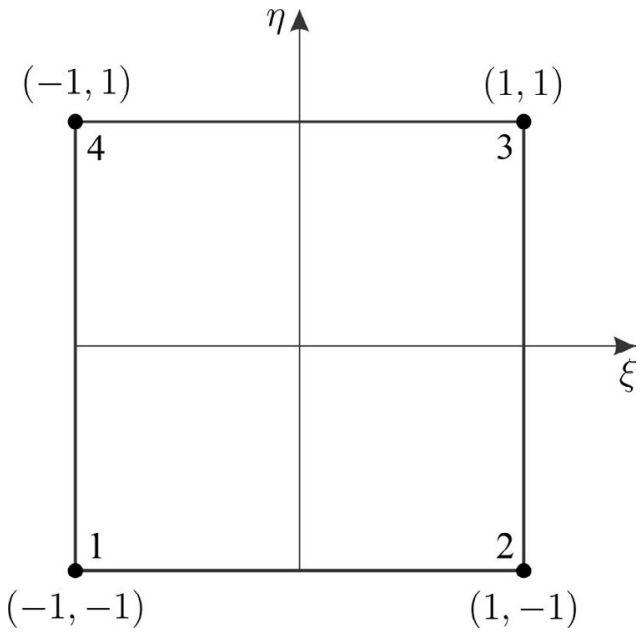

Figura 10. Elemento rectangular isoparamétrico de primer orden. Elaboración: Jaime Retama Velasco.

Las funciones de forma que permiten pasar del mundo real de la Figura 9, al mundo padre de la Figura 10, y viceversa, se expresan como:

$$
n_{i}=\frac{1}{4}\left(1+\xi \cdot \xi_{i}\right)\left(1+\eta \cdot \eta_{i}\right), \quad \forall i=1, \ldots, 4
$$

Con las funciones de forma derivadas a partir de la ecuación 12, y con las coordenadas nodales del elemento de la Figura 9, se tiene que la interpolación de la geometría, para una viga de sección variable y simétrica respecto al eje $x$, en las direcciones horizontal y vertical, está dada por:

$$
\begin{aligned}
& x(\xi, \eta)=\frac{1}{2}(1-\xi) x_{1}+\frac{1}{2}(1+\xi) x_{2} \\
& y(\xi, \eta)=\frac{1}{2}(\xi-1)(\eta) y_{1}-\frac{1}{2}(1+\xi)(\eta) y_{2}
\end{aligned}
$$

donde $x_{1}, x_{2}, y_{1} y y_{2}$ son las coordenadas nodales del elemento, Figura 9. De acuerdo con la geometría de la viga y la discretización mediante elementos finitos, se tiene que $y_{3}=-y_{2}$ y $y_{4}=-y_{2}$, lo que permite simplificar las funciones que describen la geometría.

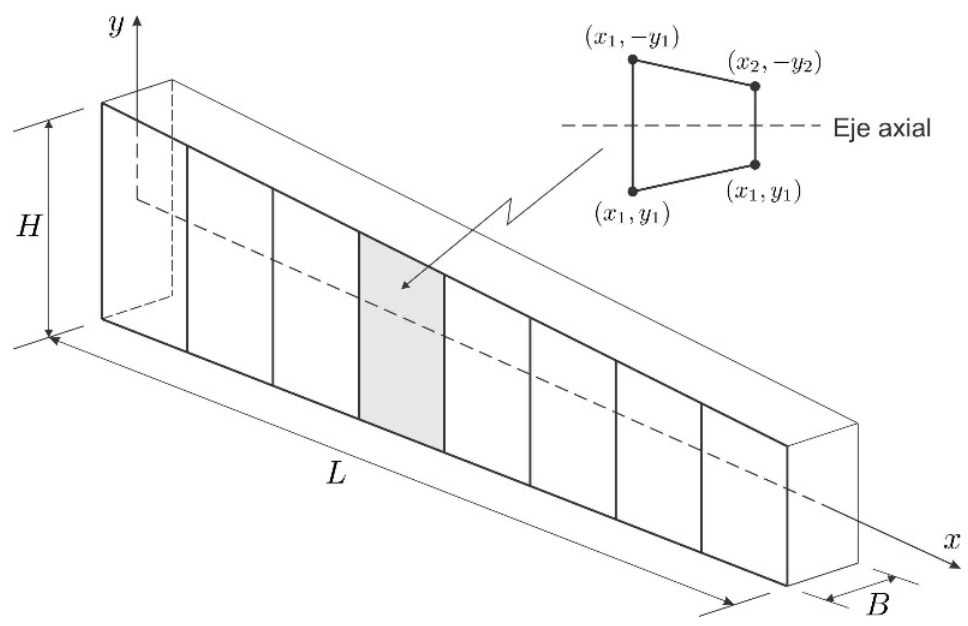

Figura 11. Viga discretizada con elementos finitos. Elaboración: Jaime Retama Velasco.

Para fines de integración, el elemento viga se debe discretizar en un número determinado de elementos finitos, que permita una integral más aproximada y evite problemas numéricos con el jacobiano de la transformación ( $\mathrm{Fi}$ gura 11). En concordancia con lo anterior, los coeficientes de flexibilidades se cambian del sistema coordenado $(x, y)$ al sistema natural $(\xi, \eta)$ por medio de: 


$$
d x d y=J d \xi d \eta
$$

donde el jacobiano $J$ se define por:

$$
\boldsymbol{J}=\frac{\partial x}{\partial \xi} \frac{\partial y}{\partial \eta}-\frac{\partial x}{\partial \eta} \frac{\partial y}{\partial \xi}
$$

Para una numeración anti-horaria de los nodos del elemento finito de la Figura 9, el jacobiano debe ser mayor o igual a cero, que en conjunto con las ecuaciones 13-15, permiten reescribir los coeficientes de flexibilidades de la viga, en función de las coordenadas locales naturales dadas en la Figura 10:

$$
\begin{aligned}
& f_{11}(\xi, \eta)=\frac{B}{E} \int_{-1}^{1} \int_{-1}^{1} \frac{[x(\xi, \eta) \cdot y(\xi, \eta)]^{2}}{I(\xi, \eta)} J d \xi d \eta \\
& f_{12}(\xi, \eta)=\frac{B}{E} \int_{-1}^{1} \int_{-1}^{1} \frac{x \cdot[y(\xi, \eta)]^{2}}{I(\xi, \eta)} J d \xi d \eta \\
& f_{22}(\xi, \eta)=\frac{B}{E} \int_{-1}^{1} \int_{-1}^{1} \frac{[y(\xi, \eta)]^{2}}{I(\xi, \eta)} J d \xi d \eta
\end{aligned}
$$

\section{Integración numérica}

Una vez realizada la transformación de los coeficientes de flexibilidades, del sistema real al sistema local natural, se procede a su integración en el mundo padre usando una regla de integración, como la de Gauss (Zienkiewicz et al., 2013; Vázquez y López, 2001); para ejemplificar la aplicación de la regla de Gauss, se utilizan dos puntos en cada dirección; sin embargo, para mejorar los resultados numéricos, se requieren más puntos de integración.

Para un coeficiente cualquiera $f(\xi, \eta)$, función dependiente de las coordenadas locales naturales, el cambio de variables al sistema local natural da por resultado:

$$
f(\xi, \eta)=\int_{-1}^{1} \int_{-1}^{1} I(\xi, \eta) J d \xi d \eta
$$

La integración numérica de la ecuación 17 se obtiene al realizar la sumatoria de la función, evaluada en cada uno de los puntos de integración de Gauss, multiplicada por los respectivos pesos:

$$
f(\xi, \eta)=\sum_{j=1}^{2}\left[\sum_{i=1}^{2} I\left(\xi_{i}, \eta_{j}\right) w_{i}\right] w_{j}
$$

En la Figura 12, se muestra la localización de los puntos de integración de Gauss en el elemento padre; esos mismos puntos de integración y sus respectivos pesos se presentan en la Tabla 1 (Vázquez y López, 2001).

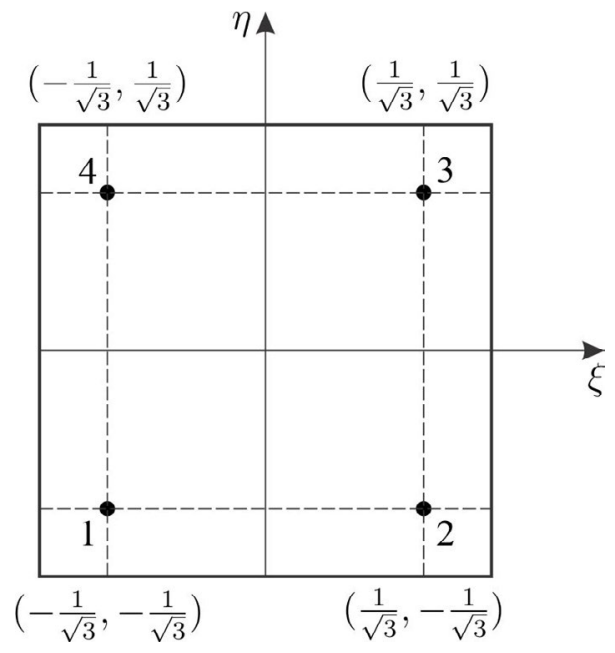

Figura 12. Dos puntos de Gauss. Elaboración: Jaime Retama Velasco.

Tabla 1. Cuadratura de Gauss de 2 por 2.

\begin{tabular}{|c|c|c|c|}
\hline Punto & $\xi_{i}$ & $\eta_{i}$ & $w_{i}$ \\
\hline 1 & $-\frac{1}{\sqrt{3}}$ & $-\frac{1}{\sqrt{3}}$ & 1.00 \\
\hline 2 & $\frac{1}{\sqrt{3}}$ & $-\frac{1}{\sqrt{3}}$ & 1.00 \\
\hline 3 & $\frac{1}{\sqrt{3}}$ & $\frac{1}{\sqrt{3}}$ & 1.00 \\
\hline 4 & $-\frac{1}{\sqrt{3}}$ & $\frac{1}{\sqrt{3}}$ & 1.00 \\
\hline
\end{tabular}

Elaboración: Jaime Retama Velasco.

La regla de Gauss permite integrar de forma exacta una función de hasta $2 n-1$ grados, donde $n$ es el número de puntos de Gauss; con dos puntos es posible integrar una función de tercer grado. Sin embargo, en el caso de la integración de los coeficientes de flexibilidades para elementos de sección variable, se tienen funciones que involucran divisiones de polinomios, por lo que se deben utilizar más puntos de integración, con el fin de minimizar el error numérico. 


\section{Ejemplo de aplicación}

Con objeto de mostrar el procedimiento de cálculo propuesto para integrar los coeficientes de flexibilidades, mediante la discretización de la sección con elementos finitos, se ejemplifica el cálculo numérico de una viga de sección variable, tipo ahusada, como la mostrada en la Figura 13. Los datos del problema son:

- Módulo de elasticidad $E=100 \frac{\mathrm{kg}}{\mathrm{cm}^{2}}$

- Relación de Poisson $\mu=0.20$

- Coeficiente de forma $\kappa=1.20$

- $H_{1}=40 \mathrm{~cm}$

- $H_{2}=20 \mathrm{~cm}$

- $L=300 \mathrm{~cm}$

- $B=15 \mathrm{~cm}$

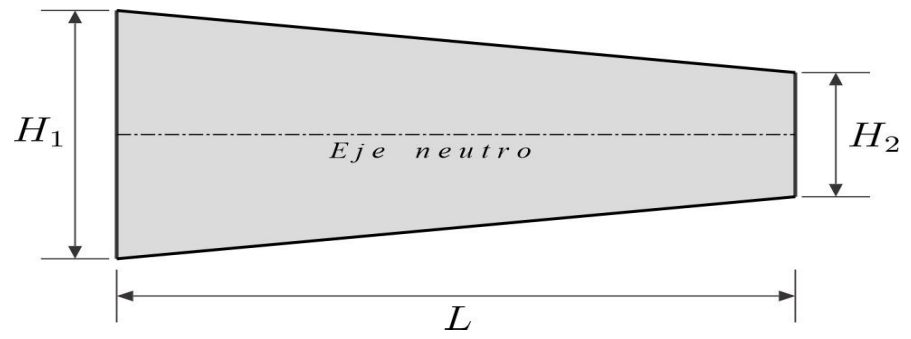

Figura 13. Viga ahusada. Elaboración: Jaime Retama Velasco.

El propósito de esta ejemplificación sólo es mostrar las ventajas de utilizar un método de integración numérica, junto con una discretización del volumen de la viga, por medio de elementos finitos. Por ello, no se consideran condiciones de carga aplicadas en la viga.

\section{Resultados}

Los resultados derivados del presente estudio se muestran en las tablas 2 y 3 ; se incluyen dos casos: uno considera la energía de deformación por flexión (teoría de Euler-Bernoulli), el otro, las energías de deformación de flexión más cortante (teoría de Timoshenko).
En la Tabla 2, se presentan los resultados obtenidos con la teoría de Euler-Bernoulli, cuando se discretiza el dominio con un máximo de cinco elementos finitos; nótese que en la última columna se incluyó como referencia la integral exacta de los coeficientes de flexibilidades; estos valores se obtuvieron con ayuda de un programa de cálculo numérico.

Tabla 2. Coeficiente de flexibilidad. Euler-Bernoulli.

\begin{tabular}{|c|c|c|c|c|c|c|}
\hline \multicolumn{6}{|l|}{ Dos puntos de integración } \\
\hline Coeficientes & 1 elemento & 2 elementos & 3 elementos & 4 elementos & 5 elementos & Exacta \\
\hline$f_{11}$ & 482.976787 & 517.232959 & 520.512274 & 521.165396 & 521.357095 & 521.50000 \\
\hline$f_{12}$ & -2.162949 & -2.240737 & -2.247884 & -2.249290 & -2.249701 & -2.25000 \\
\hline$f_{22}$ & 0.011061 & 0.011230 & 0.011246 & 0.011249 & 0.011249 & 0.01125 \\
\hline Tres puntos de integración & & & & \\
\hline$f_{11}$ & 518.918043 & 521.404296 & 521.486961 & & & 521.50000 \\
\hline$f_{12}$ & -2.244682 & -2.249814 & -2.249979 & & & -2.25000 \\
\hline$f_{22}$ & 0.011239 & 0.011250 & 0.011250 & & & 0.01125 \\
\hline
\end{tabular}

Elaboración: Jaime Retama Velasco.

En la Tabla 3, se muestran los resultados del caso en el que se aplicó la teoría de vigas de Timoshenko. Al igual que para el caso de Euler-Bernoulli, se incluyen los resultados de una integral exacta de los coeficientes de flexibilidades, obtenidos con un programa de cálculo numérico.

Tabla 3. Coeficiente de flexibilidad. Timoshenko.

\begin{tabular}{|c|c|c|c|c|c|c|}
\hline \multicolumn{7}{|c|}{ Dos puntos de integración } \\
\hline Coeficientes & 1 elemento & 2 elementos & 3 elementos & 4 elementos & 5 elementos & Exacta \\
\hline$f_{11}$ & 484.970633 & 519.229019 & 522.508495 & 523.161645 & 523.353353 & 523.493651 \\
\hline$f_{12}$ & -2.162949 & -2.240737 & -2.247884 & -2.249290 & -2.249701 & -2.250000 \\
\hline$f_{22}$ & 0.01106054 & 0.011230 & 0.011246 & 0.011249 & 0.011249 & 0.01125000 \\
\hline \multicolumn{7}{|c|}{ Tres puntos de integración } \\
\hline$f_{11}$ & 520.914234 & 523.400558 & 523.483224 & & & 523.493651 \\
\hline$f_{12}$ & -2.244682 & -2.249814 & -2.249979 & & & -2.250000 \\
\hline$f_{22}$ & 0.01123925 & 0.011250 & 0.011250 & & & 0.01125000 \\
\hline
\end{tabular}

Elaboración: Jaime Retama Velasco. 


\section{Conclusiones}

Este trabajo muestra un procedimiento para calcular de forma numérica la integral de los coeficientes de flexibilidades de un elemento viga, de sección variable, tipo ahusada o tapered. Para ello, se consideró una discretización del dominio por medio de elementos finitos rectangulares de primer orden, definidos en el plano. El método de cálculo demuestra ser viable para aplicarse a problemas en los que se tengan secciones transversales de vigas que varíen de forma compleja.

En este método, se recomienda utilizar un mínimo de tres elementos finitos, en el caso de aplicar una regla de integración de 2 puntos de Gauss, y si se usaran 3 puntos de integración, se sugiere un mínimo de dos elementos finitos. Lo anterior es válido para ambos tipos de formulaciones: Euler-Bernoulli y Timoshenko. rdp

\section{Referencias*}

Alemdar, B. N., \& White, D. W. (2005). Displacement, flexibility, and mixed beam-column finite element formulations for distributed plasticity analysis. ASCE Journal of Structural Engineering (131), 18111819. DOI: https://doi.org/10.1061/(ASCE)07332445(2005)131:12(1811)

(2011). A finite element formulation for a beam with varying cross-section geometry along its length: Flexibility-based approach. Bentley Systems Inc., Structural Engineering Group. Recuperado de https:// es.scribd.com/document/380670726/BeamElementFormulationWithVaryingSection-WhitePaper-1-pdf

Attarnejad, R., Shahba, A., \& Jandaghi, S. (2011). Analysis of non-prismatic Timoshenko beams using basic displacement functions. Advances in Structural Engineering, 2(14), 319-332. DOI: https://doi.org/10.1260/1369$\underline{4332.14 .2 .319}$

Balduzzi, G., Aminbaghai, M., Sacco, E., Fussl, J., Eberhardsteiner, J., \& Auricchio, F. (2016). Non-prismatic beams: A simple and effective Timoshenko-like model. International Journal of Solids and Structures (90), 236-250. DOI: https://doi.org/10.1016/j.ijsolstr.2016.02.017
Morganti, S., Auricchio, F., \& Reali, A. (2017). Non-prismatic Timoshenko-like beam model: Numerical solution via isogeometric collocation. Computers and Mathematics with Applications, 7(74), 1531-1541. DOI: https://doi.org/10.1016/j.camwa.2017.04.025

Banerjee, J.R., \& Williams, F. W. (1986). Exact Bernoulli-Euler static stiffness matrix for a range of tapered beam-columns. International Journal for Numerical Methods in Engineering, 9(23), 1615-1628. DOI: https:// doi.org/10.1002/nme.1620230904

Beltempo, A., Balduzzi G., Alfano, G., \& Auricchio, F. (2015). Analytical derivation of a general 2D non-prismatic beam model based on the Hellinger-Reissner principle. Engineering Structures (101), (88-98). DOI: https:// doi.org/10.1016/j.engstrcut.2015.06.020

Carrera, E., Giunta, G., \& Petrolo, M. (2011). Beam structures. Classical and advanced theories. Chichester: John Wiley \& Sons.

Eisenberger, M. (1985). Explicit Stiffness matrices for non-prismatic members. Computers and Structures, 4(20), 715-720.

(1990). An exact element method. International Journal for Numerical Methods in Engineering, 2(30, 363-370). DOI: https://doi.org/10.1002/ nme. 1620300210

(1991). Stiffness matrices for non-prismatic members including transverse shear. Computers and Structures, 4(40), 831-835. DOI: https://doi.org/10.1016/0045-7949(91)90312-A

(1994). Derivation of shape functions for exact 4-D.O.F. Timoshenko beam element. Communications in Numerical Methods in Engineering, 9(10), 673681. DOI: https://doi.org/10.1002/cnm.1640100902

Fish, J., \& Belytschko, T. (2008). A first course in finite elements. Chichester: John Wiley \& Sons.

Gallagher, R. H. (1975). Finite element analysis. Fundamentals. New Jersey: Prentice-Hall.

Ghali, A., \& Neville, A. M. (2017). Structural analysis. A unified classical and matrix approach. New York: CRC Press.

\footnotetext{
* Para una consulta óptima, se sugiere al lector que copie y pegue el vínculo en el motor de búsqueda de su preferencia.
} 
Oden, J. T., \& Ripperger, E. A. (1981). Mechanics of elastic structures. New York: Hemisphere Publishing Corporation.

Pilkey, W. D. (2002). Analysis and design of elastic beams: Computational methods. New York: John Wiley \& Sons.

Przemieniecki, J. S. (1985). Theory of matrix structural analysis. New York: Dover Publications.

Rao, S. S. (2018). The finite element method in engineering. Cambridge: Butterworth-Heinemann.

Reddy, J. N. (2019). Introduction to the finite element method. New York: McGraw-Hill.

Sack, R. L. (1994). Matrix structural analysis. Long Grove: Waveland Press.

Soltani, M. (2017). Vibration characteristics of axially loaded tapered Timoshenko beams made of functionally graded materials by the power series method. Numerical Methods in Civil Engineering Journal, 1(2), 1-14. Recuperado de http://nmce.kntu.ac.ir/article-1-103-en.html

Vázquez, M. y López, E. (2001). El método de los elementos finitos aplicado al análisis estructural. Madrid: Noela.

Veiskarami, M., \& Pourzeynali, S. (2012). Green's function for the deflection of non-prismatic simply supported beams by an analytical approach. Estonian Journal of Engineering, 18(4), 336-351. DOI: 10.3176/eng.2012.4.05

Wang, C. M., Reddy, J. N., \& Lee, K. H. (2000). Shear deformable beams and plates. Relationships with classical solutions. New York: Elsevier.

Zienkiewicz, O. C., Taylor, R. L., \& Zhu, J. Z. (2013). The finite element method. Its basis and fundamentals. New York: Butterworth-Heinemann.

Zhang, J., Zhou, Z. W., Xv, J., \& Ni, X. L. (2011). Analytical model of a single supported beam with variable section subjected to moving load. Advanced Materials Research, 291-294, 1535-1538. DOI: https://doi. org/10.4028/www.scientific.net/AMR.291-294.1535

\section{Notas de los autores:}

Jaime Retama Velasco*

Profesor de Tiempo Completo en la Facultad de Estudios Superiores Aragón, UNAM. Profesor del Programa de Especialización en Puentes de la División de Estudios de Posgrado e Investigación de la FES Aragón, UNAM. jretamav@comunidad.unam.mx

Ricardo Heras Cruz

Técnico Académico de los Laboratorios de la Licenciatura en Ingeniería Civil de la Facultad de Estudios Superiores Aragón, UNAM.

ricardo_heras@yahoo.com.mx

Agradecimientos

* El primer autor agradece el apoyo brindado para realizar la investigación a la Dirección General de Asuntos del Personal Académico (DGAPA), de la UNAM, a través del proyecto PAPIIT con número de registro IN116419. 